University of New Orleans

ScholarWorks@UNO

\title{
Dynamic and temperature effects in toggle magnetic random access memory
}

Dorin Cimpoesu

University of New Orleans

Alexandru Stancu

Leonard Spinu

University of New Orleans

Follow this and additional works at: https://scholarworks.uno.edu/phys_facpubs

Part of the Physics Commons

\section{Recommended Citation}

J. Appl. Phys. 102, 013915 (2007)

This Article is brought to you for free and open access by the Department of Physics at ScholarWorks@UNO. It has been accepted for inclusion in Physics Faculty Publications by an authorized administrator of ScholarWorks@UNO.

For more information, please contact scholarworks@uno.edu. 


\title{
Dynamic and temperature effects in toggle magnetic random access memory
}

\author{
Dorin Cimpoesua) \\ Advanced Materials Research Institute (AMRI), University of New Orleans, New Orleans, Louisiana 70148 \\ Alexandru Stancu \\ Faculty of Physics, “Al. I. Cuza” University, Iasi 700506, Romania \\ Leonard Spinu ${ }^{\text {b) }}$ \\ Advanced Materials Research Institute (AMRI), University of New Orleans, New Orleans, Louisiana 70148 \\ and Department of Physics, University of New Orleans, New Orleans, Louisiana 70148
}

(Received 22 January 2007; accepted 24 May 2007; published online 11 July 2007)

\begin{abstract}
In this paper we have studied the dynamic switching in magnetic random access memory (MRAM) and its dependence on thermal effects due to a finite temperature. The model is based on the Landau-Lifshitz-Gilbert equation and the stochastic Landau-Lifshitz-Gilbert equation which are numerically integrated. The magnetic layers are assumed to be ellipsoid shaped with each magnetic layer single domain. In addition, we have taken into account the uniaxial intrinsic anisotropy. Simulations were performed for both balanced and nonbalanced synthetic antiferromagnetic elements. The switching properties are discussed as a function of applied field pulses' length and shape. In this paper we present how the thermal fluctuations affect the switching behavior, the reliability, and the writing speed of MRAM devices. (C) 2007 American Institute of Physics. [DOI: $10.1063 / 1.2752138]$
\end{abstract}

\section{INTRODUCTION}

Magnetic random access memory (MRAM) is a promising candidate for application as a nonvolatile data storage solution because it has the potential to be fast, dense, and cost efficient with low power consumption. In toggle MRAM, proposed by Savtchenko et al., ${ }^{1}$ the MRAM cell consists of a magnetic stack with a pinned synthetic antiferromagnet (SAF) as the hard magnetic layer and a free SAF as the soft magnetic layer, where the latter is the active switching part in the cell. A SAF consists of two ferromagnetic layers coupled through a nonmagnetic layer whose thickness is tuned to provide an antiferromagnetic coupling. Switching is initiated by applying two orthogonally oriented, time delayed field pulses $\mathbf{H}_{\mathrm{W}}$ and $\mathbf{H}_{\mathrm{D}}$ (word and digit field) applied in plane at $+45^{\circ}$ and $-45^{\circ}$ with respect to the inplane easy axis of the element. A disadvantage of this scheme is that the switching process is a toggle process, i.e., for writing a bit a preread step is necessary, which increases the access time. Fujiwara et al. $^{2-4}$ and Worledge ${ }^{5}$ investigated the magnetization response of the SAF bilayer system to the applied field assuming that the magnetic moments are constrained to lie in the layer's plane, and neglecting precessional effects during reversal, i.e., that the magnetization lie in a minimum of the free energy at any moment. To reduce the writing field, Engel et al. ${ }^{6}$ proposed to apply a magnetic field in the easy axis direction. The simplest way to do this is to generate a bias field from the unbalanced pinned layers of magnetic tunneling junction.

The previous descriptions ${ }^{1-5}$ of the Savtchenko switch-

\footnotetext{
a)Permanent address: “Al. I. Cuza” University, Faculty of Physics, Iasi 700506, Romania; electronic mail: cdorin@uaic.ro

${ }^{b)}$ Electronic mail: 1spinu@uno.edu
}

ing scheme are restricted to the quasistatic regime, i.e., the switching takes the form of a Stoner-Wohlfarth rotation and does not take into account the dynamics of the magnetization. As the technological demand pushes for lower access time, the study of dynamics of the moments in a MRAM cell becomes indispensable. A dynamic simulation of toggle MRAM operating field, both at $T=0 \mathrm{~K}$ and room temperature is presented in Ref. 7.

When the fields are applied in short and fast rising pulses, the switching is dominated by the precession torque, leading to a so-called precessional dynamics. It is remarkable that the precessional reversal can provide a switching field, below Stoner-Wohlfarth limit. ${ }^{8-12}$ Numerical studies have shown that the switching time can be reduced by suppressing the ringing effect, so that the magnetization moves along a so-called ballistic trajectory. A fast (subnanosecond) reversal mode proved to be possible with a combination of transverse and longitudinal pulsed fields. ${ }^{13}$ The method requires precise control of the pulse amplitude and duration, so that the system is already in equilibrium at the end of the field pulses, and no magnetic ringing occurs. An ultrafast direct writing scheme with unipolar field pulses, which allows the back switching by reversing the temporal sequence of the two pulses, is presented in Ref. 14 .

With memory area density increasing and the memory cell size further shrinking, the study of thermal fluctuations in MRAM structures becomes extremely important in recording thermal stability. This study is motivated also by considerable interest in thermally assisted writing of MRAM, which might further increase memory density, while maintaining long data retention. 


\section{THE MODEL}

The model we used in the simulations is based on Landau-Lifshitz-Gilbert (LLG) equation ${ }^{15}$ and stochastic Landau-Lifshitz-Gilbert (SLLG) equation ${ }^{16}$ assuming that each magnetic layer is a single domain. In the presence of thermal agitation it is supposed that the dissipative term in LLG equation describes only the statistical (ensemble) average of rapidly fluctuating random forces, and that for an individual particle a stochastic thermal field should be added. The stochastic field changes the deterministic motion of the magnetization into a random walk. The SLLG equation is numerically integrated using an implicit midpoint timeintegration technique. ${ }^{17}$ No temperature dependence of the anisotropy constant and saturation magnetization is taken. The magnetic properties follow from an average over many numerical realizations of the dynamic process (discretized Brownian paths).

For the switching process we considered only the soft SAF stack of a MRAM cell, and assumed that the two ferromagnetic layers are identical except for their thickness. As ferromagnetic material, Permalloy with saturation magnetization $4 \pi M_{S}=10.8 \mathrm{kOe}$ was chosen. The phenomenological Gilbert damping constant $\alpha=0.008$ (Refs. 18 and 19) was used throughout the paper, unless otherwise specified. The damping parameter is treated as a constant, even though there are theoretical and experimental evidences that $\alpha$ can be affected with various factors such as the magnetization's orientation $^{20,21}$ and temperature. ${ }^{22}$

The magnetic layers are assumed to be ellipsoids making the demagnetizing field uniform across the entire layer. The ellipsoid's principal axes are taken along $x, y$, and $z$ : longaxis length of $120 \mathrm{~nm}$ (along $O_{x}$ axis) and short-axis length of $100 \mathrm{~nm}$ (along $O_{y}$ axis). The thickness of bottom layer (considered to be adjacent to the tunnel junction and determining the state of the cell memory) is fixed at $t_{1}=5 \mathrm{~nm}$, leading to the demagnetizing fields: $H_{D, x}=N_{x} M_{S}=318.8 \mathrm{Oe}$, $H_{D, y}=N_{y} M_{S}=471 \mathrm{Oe}$, and $H_{D, z}=N_{z} M_{S}=10.06 \mathrm{kOe},{ }^{23}$ and to in-plane shape-anisotropy field $H_{\mathrm{sh}}=\left(N_{y}-N_{x}\right) M_{S}=98.2 \mathrm{Oe}$, where $N_{x}, N_{y}$, and $N_{z}$ are the corresponding demagnetizing factors. The thickness $t_{2}$ of the top layer is varied so that $t=t_{2} / t_{1} \leqslant 1$ (Ref. 1). In addition we take into account a uniaxial intrinsic anisotropy $H_{\mathrm{Ku}}=10$ Oe along the $O_{x}$ axis. The state " 1 " of the MRAM cell is defined as $\mathbf{m}_{1}=-\hat{\mathbf{x}}, \mathbf{m}_{2}$ $=\hat{\mathbf{x}}$, and the state " 0 " as $\mathbf{m}_{1}=\hat{\mathbf{x}}, \mathbf{m}_{2}=-\hat{\mathbf{x}}$.

Because an instantaneous change of the applied field from zero to some other value is not very realistic, sinusoidal time dependence for the field pulse rise and fall are assumed. The rise/fall time is a function of the pulse's amplitude so that the field sweep rate $v_{H}$, defined as the ratio between the amplitude and pulse rise/fall time, is constant. The field notation

pulses with a width $T_{W}=T_{D}=T_{H}$ (the amount of time the pulse takes to go from zero to high and back to zero again) are delayed against each other with $\Delta T_{\mathrm{WD}}=T_{W} / 2$ offset and $\Delta T_{\mathrm{WD}}$ overlap. The pulse sequences are sketched in Fig. 1. The final state is taken at $\Delta T_{\mathrm{WD}}$ after the termination of digit field pulse.

The effective fields for the two layers consist of applied

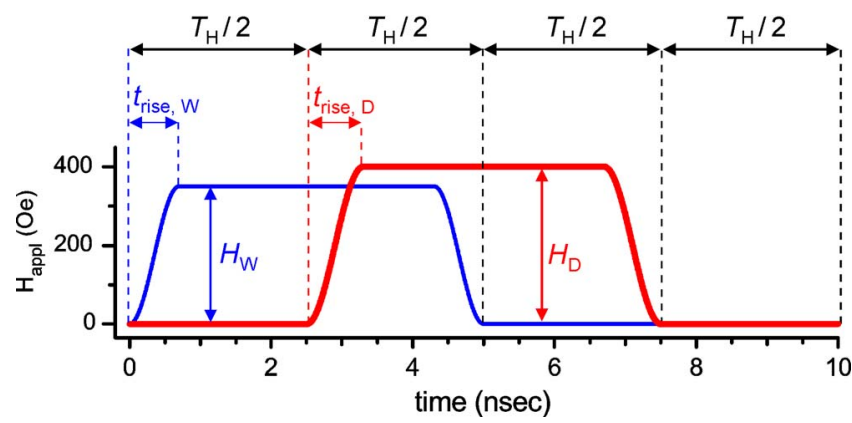

FIG. 1. (Color online) Time dependence of the applied field pulses. The field sweep rate is defined as $v_{H}=H_{\mathrm{W}} / t_{\text {rise, } \mathrm{W}}=H_{\mathrm{D}} / t_{\text {rise, } \mathrm{D}}$ and it is constant.

field, uniaxial anisotropy field, demagnetizing field, antiferromagnetic coupling between the two layers $J$, and thermal field

$$
\begin{aligned}
\mathbf{H}_{1,2}^{\mathrm{eff}}= & \mathbf{H}_{\mathrm{appl}}+H_{K u}\left(\mathbf{m}_{1,2} \cdot \hat{\mathbf{x}}\right) \hat{\mathbf{x}}-M_{S} \stackrel{\mathbf{N}}{1,2}_{1,2} \cdot \mathbf{m}_{1,2} \\
& -\frac{J}{M_{S} t_{1,2}} \mathbf{m}_{2,1}+\mathbf{H}_{1,2}^{\mathrm{th}},
\end{aligned}
$$

where $\mathbf{m}_{i}=\mathbf{M}_{i} / M_{S}$ is the unit vector describing the magnetization orientation of the two ferromagnetic layers $(i=1,2)$, and $\overleftrightarrow{\mathbf{N}}_{i}$ is the demagnetizing tensor where the only nonvanishing components are the diagonal elements. For the sake of simplicity the dipolar interactions between the two layers are not taken into account because, similar to coupling constant $J$, these interactions also favor an antiparallel alignment. However, we mention that due to the elliptical shape of the layers the dipolar fields have an asymmetry in the spatial dependence, whereas $J$ has none. ${ }^{24}$

The time evolution of magnetization is found by integrating the coupled LLG (or SLLG) equations,

$$
\frac{d \mathbf{M}_{i}}{d t}=-\gamma \mathbf{M}_{i} \times \mathbf{H}_{i}^{\mathrm{eff}}-\frac{\alpha \gamma}{M_{s}} \mathbf{M}_{i} \times\left(\mathbf{M}_{i} \times \mathbf{H}_{i}^{\mathrm{eff}}\right),
$$

where $\gamma=\left|\gamma_{0}\right| /\left(1+\alpha^{2}\right), \quad\left|\gamma_{0}\right|=2.211 \times 10^{5}(\mathrm{rad} / \mathrm{s}) /(\mathrm{A} / \mathrm{m})$ is the gyromagnetic ratio.

\section{RESULTS}

\section{A. Dynamic effects in MRAM}

The results from Refs. 2-5 are obtained in the static case, assuming that the magnetic moments are constrained to lie in the $x y$ plane during their reversal, due to the large shape anisotropy created by the small thickness-to-width ratio, and that the magnetization reversal mechanism relies on quasistatic energy mechanism such that the magnetization lies in a minimum of the free energy at any moment. However, as is shown in the dynamical equation (2), the first term on the right-hand side will pull the magnetization vectors away from the xy plane. Initially, when the two magnetizations vectors $\mathbf{M}_{1}$ and $\mathbf{M}_{2}$ are aligned along the $x$ axis (easy axis), the $y$ component of the applied field exerts a torque which causes a rotation of $\mathbf{M}_{1}$ and $\mathbf{M}_{2}$ out of the film plane, in the $x z$ plane. Then, the demagnetizing field rotates $\mathbf{M}_{1}$ and $\mathbf{M}_{2}$ toward each other, with a larger precession for the magnetization which initially was aligned along the $-x$ direction 
because its effective field has a larger $y$ component. Thus, the assumption that the magnetization moves in the $x y$ plane is true only when the damping constant $\alpha$ is sufficiently large so that the first term on the right-hand side of Eq. (2) (which describes the precession) can be neglected with regard to the second term (which describes the damping), and the magnetization moves along the energy-steepest-descent path. In a realistic system $\alpha$ is not infinitely large and the magnetization does not move along the energy-steepest-descent path. On the other hand, in general, a path from one minimum to the other passes over a saddle point. If the applied field has a rise time shorter than the energy relaxation time it is possible that the initial magnetization to be at a higher energy than the energy at the saddle point, and the system switches at a smaller field than in the static case. For very large $\alpha$ the system evolves along the energy-steepest-descent path into the minimum near the initial state, while for small $\alpha$ the precessional term can drive the magnetization across a large range of motion around the energy minima before energy is dissipated, and fall into one of them as a function of the balance between the energy gained from the applied field and the energy dissipated during the motion. For very large $\alpha$, the system switches if the basin of attraction of the initial state shrinks to a point, while for small $\alpha$, the system can switch if the system reaches the basin of the final state.

On the other hand, the magnetization reversal in a time dependent applied field is different from that in a constant field because the energy change rate depends on the applied field sweep rate,

$$
\begin{aligned}
\frac{d W}{d t}= & -\frac{\gamma}{M_{S}} \frac{\alpha}{1+\alpha^{2}}\left[t_{1}\left(\mathbf{M}_{1} \times \mathbf{H}_{1}^{\mathrm{eff}}\right)^{2}+t_{2}\left(\mathbf{M}_{2} \times \mathbf{H}_{2}^{\mathrm{eff}}\right)^{2}\right] \\
& -\left(t_{1} \mathbf{M}_{1}+t_{2} \mathbf{M}_{2}\right) \cdot \frac{d \mathbf{H}_{\mathrm{appl}}}{d t},
\end{aligned}
$$

where $W$ is energy density per unit area. The last term in Eq. (3) can be either positive or negative depending on the relative direction of $\mathbf{M}_{1}, \mathbf{M}_{2}$, and $d \mathbf{H}_{\text {appl }} / d t$.

In order to determine the magnetic field pulse's parameters where fast and stable MRAM operation can be achieved, we have studied the switching properties as a function of applied field pulses' length and shape and also as a function of the exchange antiferromagnetic coupling between the two ferromagnetic layers.

The switching behavior of a balanced and a nonbalanced SAF element at $T=0 \mathrm{~K}$, as a function of the applied digit and word field pulse strengths for different values of the applied field sweep rate $v_{H}$ is presented in Fig. 2. The pulses' width is $T_{H}=5 \mathrm{~ns}$, and the antiferromagnetic coupling constant is $h_{J}=H_{J} /\left(H_{\mathrm{Ku}}+H_{\mathrm{sh}}\right)=4$, where $H_{J}$ is the effective coupling exchange field. The interlayer coupling thus considered is of the same order of magnitude as the applied field. For a balanced element, due to the symmetry, only nonswitching (black areas) or toggle mode (white areas) can be obtained. For toggle mode the state 1 is switched to a 0 state after field pulse sequence, and repeating the field pulse sequence on the stored 0 state returns it to a 1 . Toggle mode only results in magnetization switching without being able to choose the final state. Thus, to be able to write the memory element into

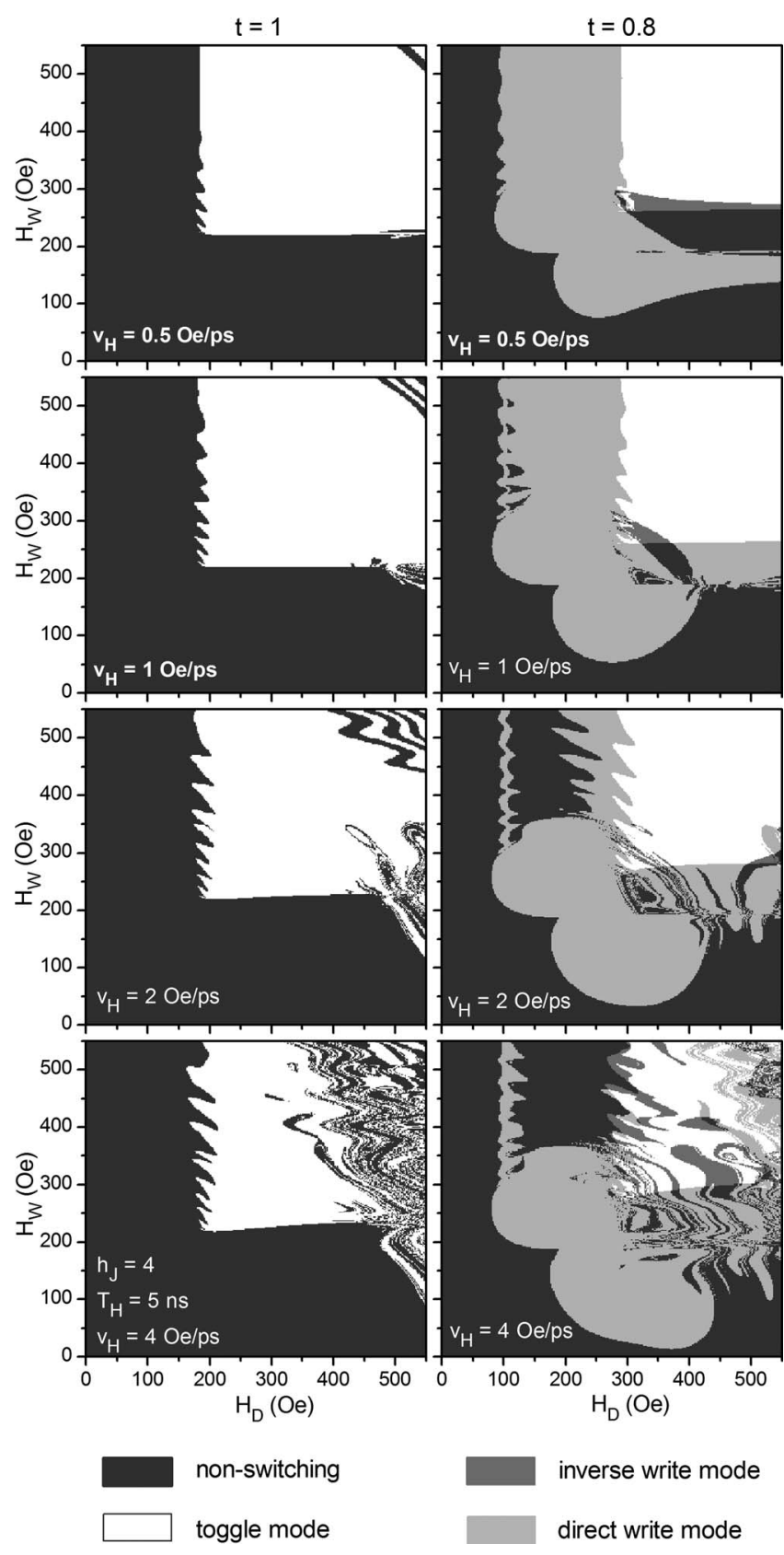

FIG. 2. Switching diagram of a balanced (left) and a nonbalanced (right) SAF element, at $T=0 \mathrm{~K}$, as a function of the applied field pulse strengths for different values of the applied field sweep rate $v_{H}$. The antiferromagnetic coupling constant is $h_{J}=4$ and the pulse width $T_{H}=5 \mathrm{~ns}$. Black areas represent nonswitching, light-gray areas direct write, dark-gray areas inverse write, and white areas toggle mode.

the desired state, the initial state of MRAM must first be read and compared to the state to be written. For a nonbalanced element besides nonswitching and toggle modes, the direct write mode is in effect (light-gray areas in Fig. 2). For direct writing the state 1 is switched to a 0 state after field pulse sequence, but the state 0 is not switched. When using the direct write method there is no need to determine the initial state of the MRAM cell because the state is switched only if the state being written is different from the stored state. Besides these three modes, nonswitching, toggle, and direct write, described in Ref. 1, we have identified a different write mode that we called inverse write mode (dark-gray 

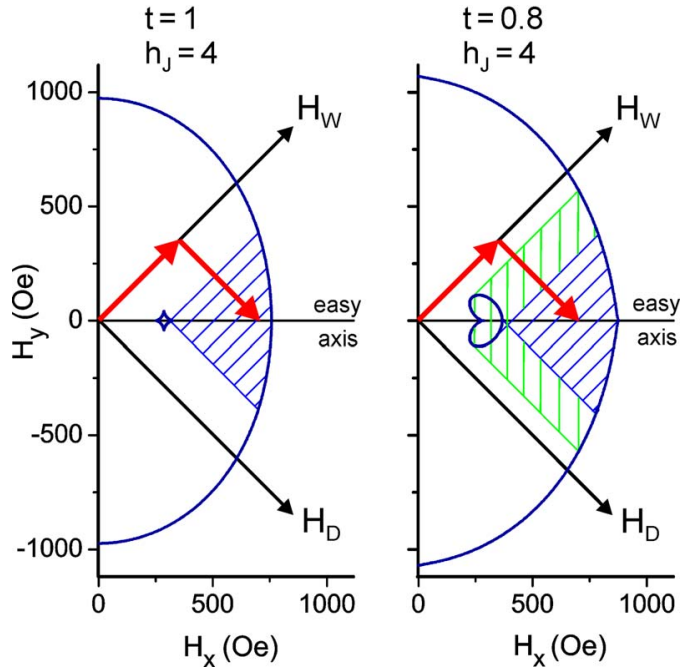

FIG. 3. (Color online) Critical field curves obtained by Fujiwara et al. (Refs. 2-4) for $h_{J}=4$, and for $t=1$ (left) and $t=0.8$ (right), with the operating field margins for the toggle mode (the area hatched by the inclined lines) and the direct write mode (the area hatched by the vertical lines). The amplitude of the word and digit field showed in the figures is $H_{\mathrm{W}}=H_{\mathrm{D}}=500 \mathrm{Oe}$.

areas in Fig. 2) for which the state 0 is switched to a 1 state after field pulse sequence, but the state 1 is not switched. This mode is strongly affected by the dynamic switching of the magnetization.

Conditions for direct/toggle mode are obtained by Fujiwara $e t a l .^{2-4}$ using the critical field curves. Critical curves and the operating field margins for the toggle mode (the area hatched by the inclined lines) and the direct write mode for our system are sketched in the Fig. 3 .

From Fig. 2 we can see that the final state is sensitive to the sweep rate $v_{H}$ of the applied field pulses, and that the switching behavior shares similar features with the precession dominated dynamics of a single Stoner-Wohlfarth particle presented in Ref. 9 and for two interacting StonerWohlfarth particles in Ref. 25. The boundary which delimits the toggle mode along $H_{\mathrm{D}}$ and $H_{\mathrm{W}}$ axes is not smooth, and the switching and nonswitching areas alternate with increasing field amplitude. As the field sweep rate increases the extent of the instability region is also increasing. Compared to the results for $v_{H}=0.5 \mathrm{Oe} / \mathrm{ps}$, we observe at $v_{H}=4 \mathrm{Oe} / \mathrm{ps}$ a strongly alternating behavior in the upper right part of the diagram. This alternating behavior has two causes: If the pulse's length is too short a significant ringing of the magnetization still exists during the field pulse and the final state is determined by the position of the magnetization at the end of the pulse. On the other hand, we have found that for low values of damping constant $\alpha$, the basins of attraction of fixed points have a layerlike structure, similar with the case of a single Stoner-Wohlfarth particle. ${ }^{12}$ In order to minimize the first cause we have made simulations with longer field pulses (see Fig. 4). The diagrams reflect a higher stability of the switching process along the $H_{\mathrm{D}}$ and $H_{\mathrm{W}}$ axes but still show an alternating behavior in the upper right part. As we can see in Fig. 4(b), the two magnetization vectors switch from a fixed point, followed by a few ringing periods. For a fast and reliable operation such ringing must be avoided as the damping time can take several nanoseconds. The ringing

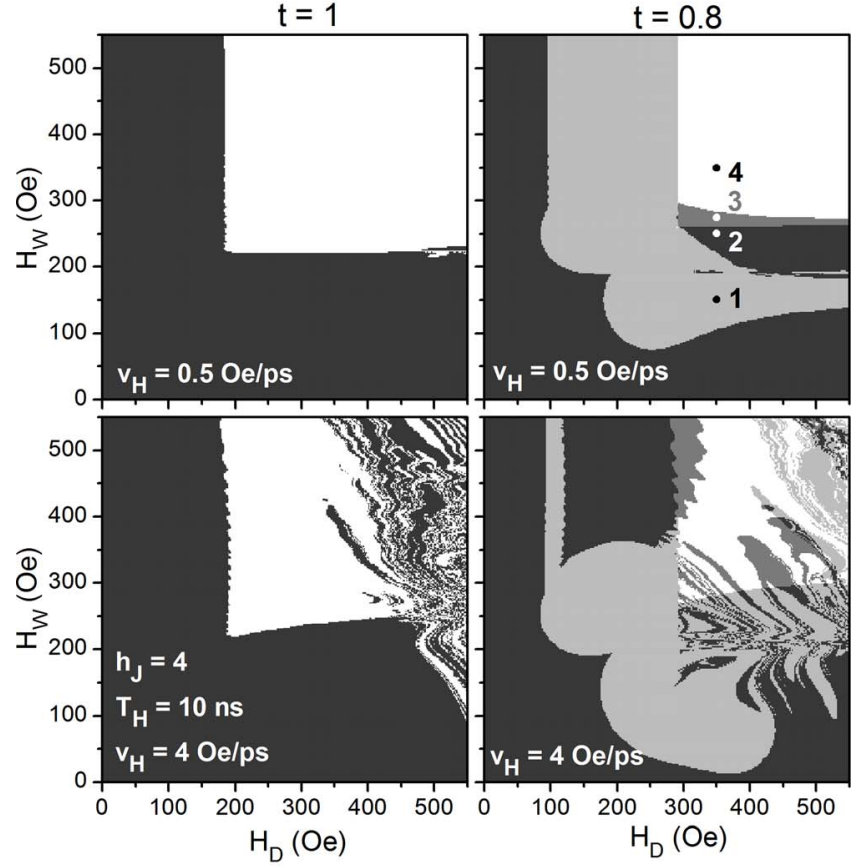

(a)

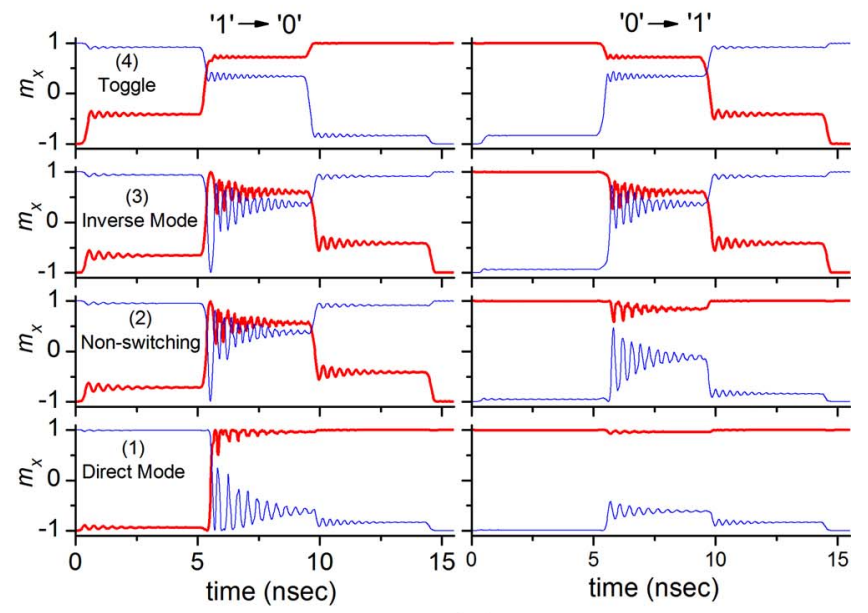

(b)

FIG. 4. (Color online) (a) Switching diagram of a balanced (left) and a nonbalanced (right) SAF element for $T=0 \mathrm{~K}, h_{J}=4, T_{H}=10 \mathrm{~ns}$, and two values of the field sweep rate: $v_{H}=0.5 \mathrm{Oe} / \mathrm{ps}$ and $v_{H}=4 \mathrm{Oe} / \mathrm{ps}$. (b) Time evolution of $m_{1, x}$ (bold line) and $m_{2, x}$ (thin line) when the initial magnetization state is "1" (left) and "0" (right), at the positions indicated in the second switching diagram.

can be avoided either by a precise control of a pulse amplitude/duration and MRAM cell parameters so that the system moves along a ballistic trajectory, or using a relatively slow rise time pulse. The experimentally reported pulse width in an MRAM circuit is around $10 \mathrm{~ns}$ with a rise time of around $1 \mathrm{~ns}^{26,27}$

An increase of the exchange antiferromagnetic coupling between the two ferromagnetic layers leads to an increase of the stable switching region but, at the same time, a larger operating field is required (see Fig. 5).

In all the previous presented cases we observe that for a nonbalanced element the direct write mode boundary is 

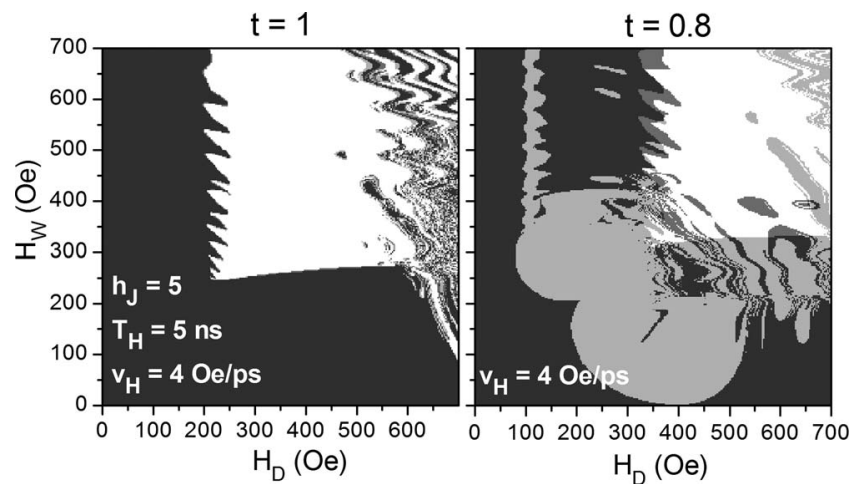

FIG. 5. Switching diagram of a balanced (left) and a nonbalanced (right) SAF element for $T=0 \mathrm{~K}, h_{J}=5, T_{H}=5 \mathrm{~ns}$, and $v_{H}=4 \mathrm{Oe} / \mathrm{ps}$.

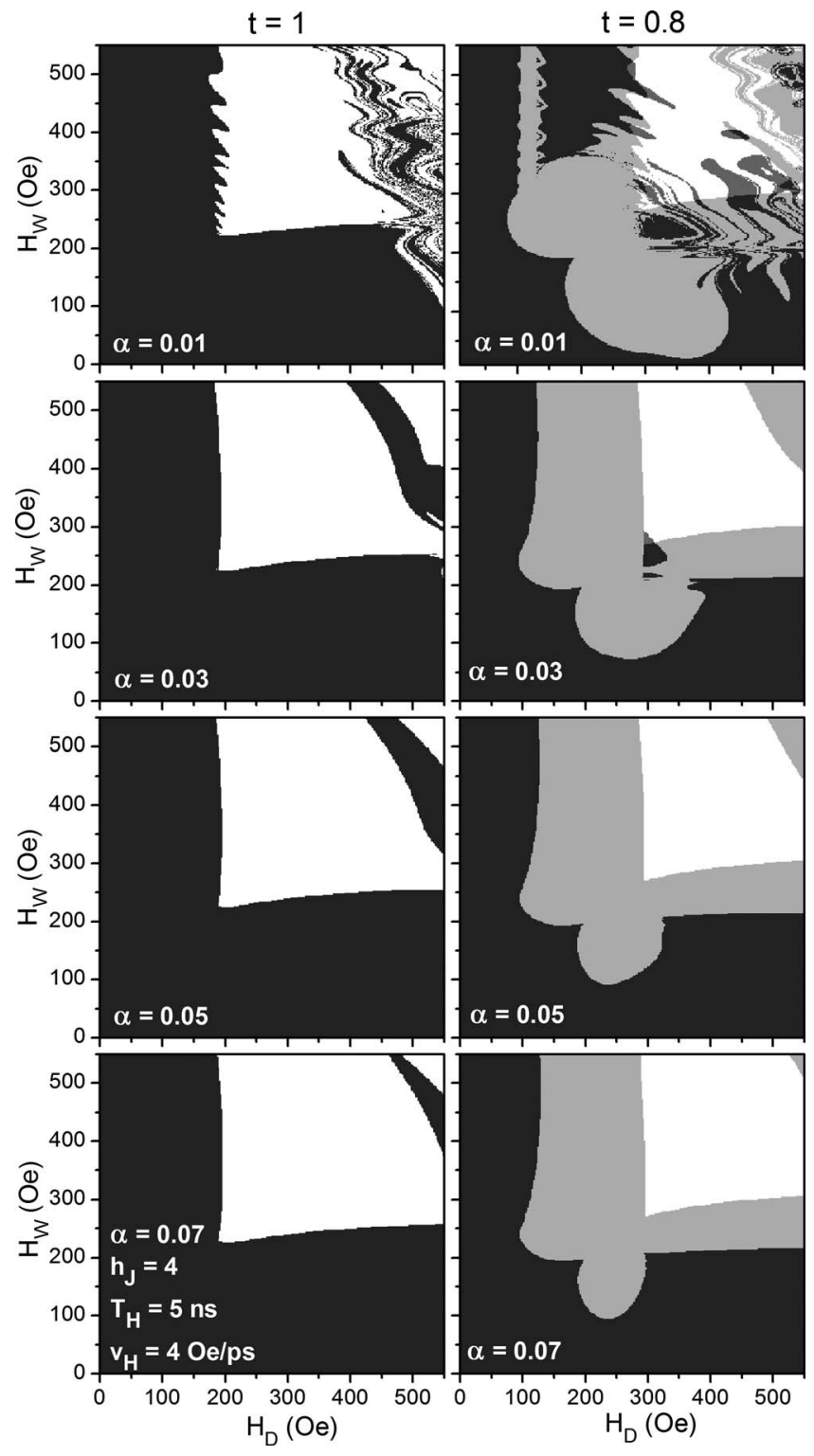

FIG. 6. Switching diagram of a balanced (left) and a non-balanced (right) SAF element for $T=0 \mathrm{~K}, h_{J}=4, T_{H}=5 \mathrm{~ns}, v_{H}=4 \mathrm{Oe} / \mathrm{ps}$, and different values of the damping constant $\alpha$.

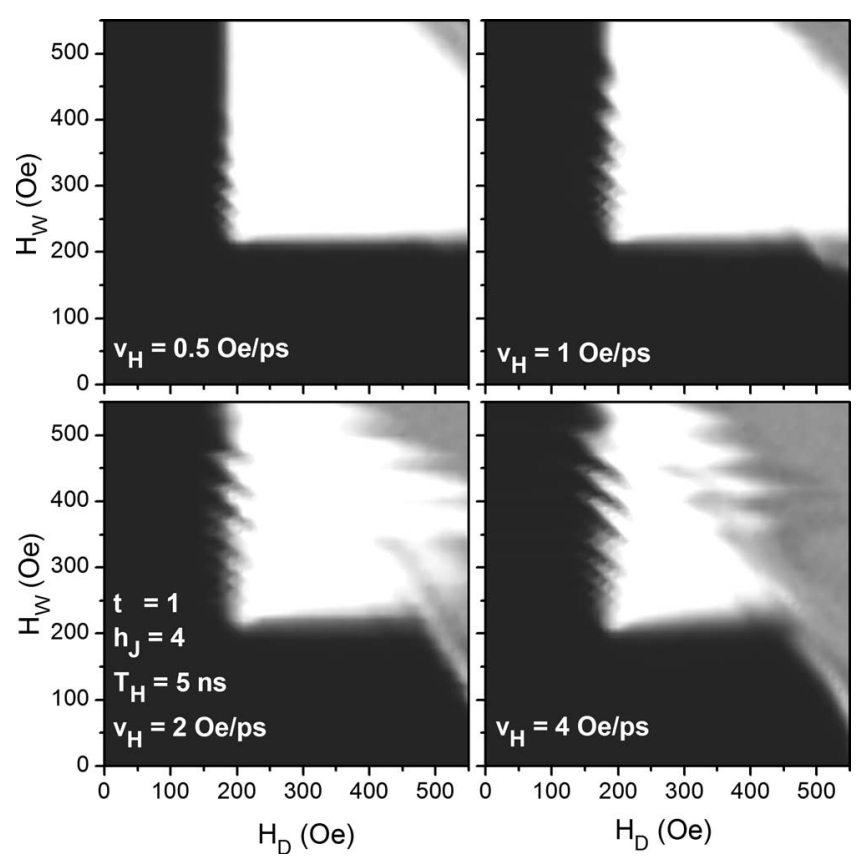

FIG. 7. Probability of switching of a balanced SAF element for $T=300 \mathrm{~K}$, $h_{J}=4, T_{H}=5 \mathrm{~ns}$, and different values of the applied field sweep rate $v_{H}$. Black areas represent nonswitching, i.e., switching probability $P=0$, white areas $P=1$ (toggle mode), and the intermediate values of switching probability are represented with shades of gray.

shifted toward the $H_{\mathrm{D}}$ axis as the field sweep rate is increased, which reflects the instability of the switching process.

Recent studies ${ }^{28-30}$ for ultrathin films show that the damping constant $\alpha$ is enhanced when a nonmagnetic metal is deposited on the ferromagnetic film. Our simulations show that an increase of $\alpha$ diminishes the layer structure of the switching diagrams and gives rise to a clearer separation between the nonswitching/direct mode and toggle mode (see Fig. 6). An increase of $\alpha$ increases the energy dissipation rate [see Eq. (3)] and consequently diminishes the ringing of the magnetization.

\section{B. Thermal relaxation effect}

As MRAM device sizes continue to shrink and operating frequencies continue to increase, high-frequency magnetic noise due to the thermal fluctuations may pose a fundamental limitation to MRAM performance. The magnetic moments effect random oscillations by thermal activation close to the bottom of the energy minima, but also fluctuations can allow the system to be thermally activated over finite energy barriers.

Assuming that from $N_{0}$ MRAM cells, only one is allowed to lose memory during the lifetime $\tau_{\text {life }}$, and that the thermal switching occurs simultaneously for both layers, Fujiwara $e t a l .^{2}$ obtained, for a balanced SAF in zero applied field,

$$
\Delta E=2\left(K_{\mathrm{u}}+K_{\mathrm{sh}}\right) V \geqslant k_{B} T \ln \left(f_{0} N_{0} \tau_{\text {life }}\right),
$$

where $V$ is the volume of each layer, $k_{B}$ is Boltzmann's constant, and $f_{0}$ is the attempt frequency. Assuming $f_{0}=10^{9} \mathrm{~Hz}$, $N_{0}=10^{12}$, and $\tau_{\text {life }}=10 \mathrm{yr}, \Delta E / k_{B} T \geqslant 68$ is obtained. In our 

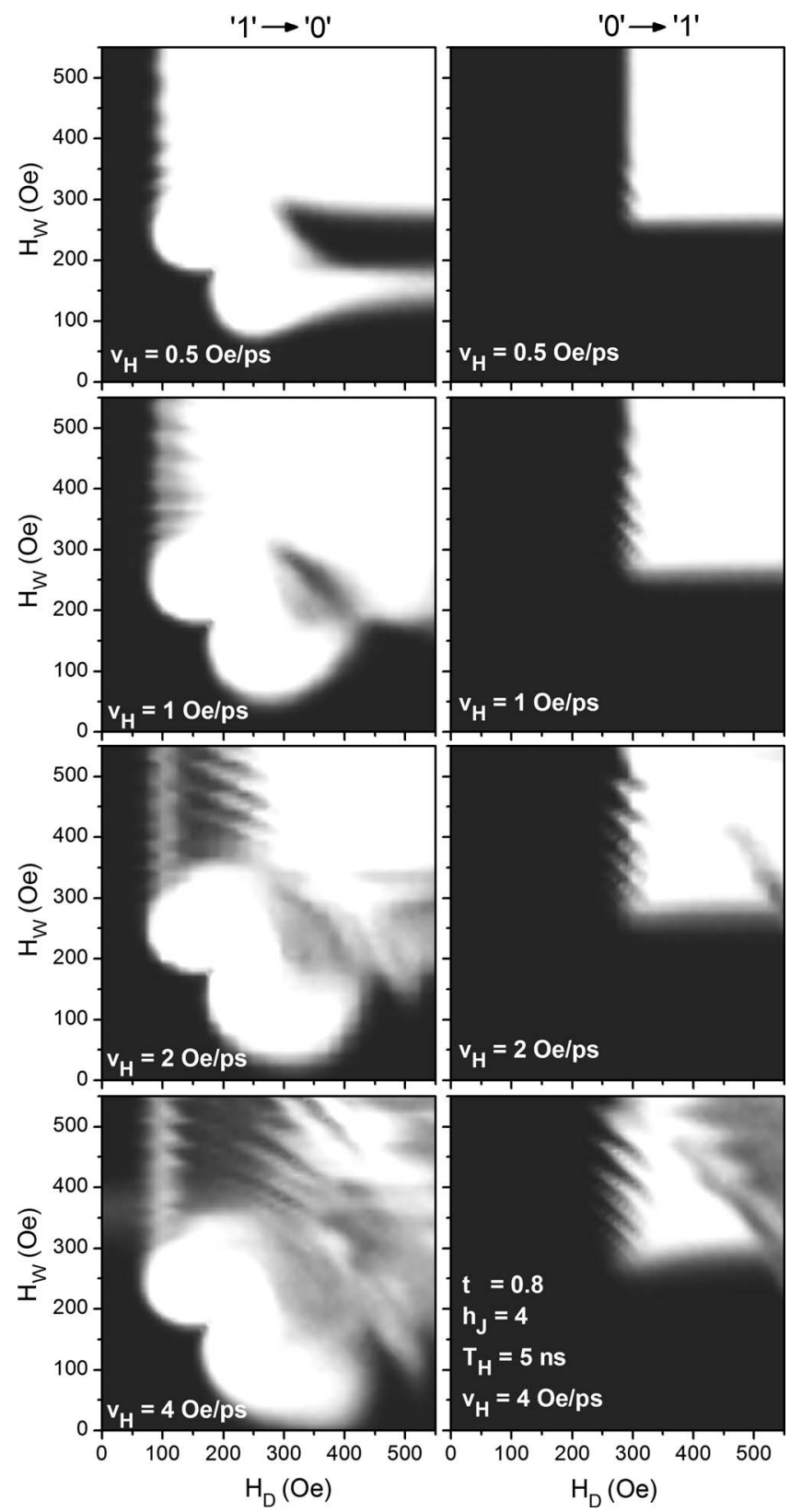

FIG. 8. Probability of switching of a nonbalanced SAF element when the initial magnetization state is ' 1 ' (left) and ' 0 ' (right), for $T=300 \mathrm{~K}, h_{J}=4$, $T_{H}=5 \mathrm{~ns}$, and different values of the applied field sweep rate $v_{H}$. Black areas represent nonswitching, i.e., switching probability $P=0$, white areas $P=1$, and the intermediate values of switching probability are represented with shades of gray.

simulations, $\Delta E / k_{B} T=70.5$. The probability of switching is plotted in a gray coded diagram as a function of the applied digit and word field pulse strengths in Figs. 7 and 8 for different values of the applied field sweep rate $v_{H}$. Each simulated point from diagrams represents the statistic of 1024 repeatedly writing operations at certain $H_{\mathrm{D}}$ and $H_{\mathrm{W}}$. We can see that instead of a clear border between switching and nonswitching areas we have a transition region where the final state is sensitive to thermal fluctuations, even if the SAF's parameters were chosen so that the condition from Eq. (4) is fulfilled. This can be a consequence of the magnetization vector trajectories which do not lie in the layer planes and can provide a lower switching field, below the static limit, as we have shown previously.

\section{CONCLUSIONS}

Based on the magnetization vector dynamics, as described by LLG/SLLG equation of motion, the switching properties of a MRAM cell have been presented. We have shown that besides nonswitching, toggle, and direct write mode, ${ }^{1}$ an inverse write mode can be identified. In dealing with the problem of improving the writing time, one needs to pay careful attention to the parameters describing the pulse shape. As the applied field sweep rate $v_{H}$ rises, the switching diagrams increasingly have layerlike structures with switching/nonswitching areas. The extent of the instability region is increasing with $v_{H}$. This alternating behavior has two causes: the ringing of the magnetization during the field and the layerlike structure of basins of attraction of fixed points. A reliable switching is obtained only if the ringing is avoided either by a precise control of the pulse and MRAM cell parameters, so that the system moves along a ballistic trajectory, or using a relatively slow rise time pulse. Also, we have presented how the thermal fluctuations affect the switching behavior.

\section{ACKNOWLEDGMENTS}

Work at AMRI was supported by DARPA under Grant No. HR0011-05-1-0031. This work was partially supported by Romanian CNCSIS under the Grant A(RELSWITCH).

${ }^{1}$ L. Savtchenko, B. N. Engel, N. D. Rizzo, M. F. Deherrera, and J. A. Janesky, U.S. Patent 6,545,906 B1 (April 8, 2003).

${ }^{2}$ F. H. Fujiwara, S. Y. Wang, and M. Sun, Trans. Magn. Soc. Jpn. 4, 121 (2004).

${ }^{3}$ F. H. Fujiwara, S. Y. Wang, and M. Sun, J. Appl. Phys. 97, 10 P507 (2005).

${ }^{4}$ S. Y. Wang and F. H. Fujiwara, J. Appl. Phys. 98, 024510 (2005).

${ }^{5}$ D. C. Worledge, IBM J. Res. Dev. 50, 69 (2006).

${ }^{6}$ B. N. Engel, J. A. Janesky, and N. D. Rizzo, U.S. Patent No. 6,633,498 B1 (October 14, 2003).

${ }^{7}$ S. Wang, H. Fujiwara, J. Dou, Z. Li, and Y. Huai, IEEE Trans. Magn. 43, 2337 (2007)

${ }^{8}$ L. He, W. D. Doyle, and H. Fujiwara, IEEE Trans. Magn. 30, 4086 (1994).

${ }^{9}$ M. Bauer, J. Fassbender, H. Hillebrands, and R. L. Stamps, Phys. Rev. B 61, 3410 (2000).

${ }^{10}$ J. Miltat, G. Albuquerque, and A. Thiaville, in Spin Dynamics in Confined Magnetic Structures I, edited by B. Hillebrands and K. Ounadjela (Springer-Verlag, Berlin, 2001).

${ }^{11}$ K. Z. Gao, E. D. Boerner, and H. N. Bertram, J. Appl. Phys. 93, 6549 (2003).

${ }^{12}$ Z. Z. Sun and X. R. Wang, Phys. Rev. B 71, 174430 (2005).

${ }^{13}$ J. V. Kim, T. Devolder, C. Chappert, C. Maufront, and R. Fournel, Appl. Phys. Lett. 85, 4094 (2004).

${ }^{14}$ H. T. Nembach, C. Bayer, H. Schulthesis, M. C. Weber, P. M. Pimentel, P. A. Beck, B. Leven, and R. B. Hillebrands, Appl. Phys. Lett. 87, 142503 (2005).

${ }^{15}$ T. L. Gilbert, Phys. Rev. 100, 1243 (1955).

${ }^{16}$ W. F. Brown, Jr., Phys. Rev. 130, 1677 (1963).

${ }^{17}$ M. d'Aquino, C. Serpico, G. Coppola, I. D. Mayergoyz, and G. Bertotti, J. Appl. Phys. 99, 08B905 (2006).

${ }^{18}$ C. E. Patton, Z. Frait, and C. H. Wilts, J. Appl. Phys. 46, 5002 (1975).

${ }^{19}$ M. R. Freeman, W. Hiebert, and A. Stankiewicz, J. Appl. Phys. 83, 6217 (1998).

${ }^{20}$ C. E. Patton, J. Appl. Phys. 39, 3060 (1968).

${ }^{21}$ R. D. McMichael, M. D. Stiles, P. J. Chen, and W. F. Egelhoff, Jr., J. Appl. Phys. 83, 7037 (1998).

${ }^{22}$ C. D. Stanciu, A. V. Kimel, F. Hansteen, A. Tsukamoto, A. Itoh, A. 
Kirilyuk, and Th. Rasing, Phys. Rev. B 73, 220402(R) (2006).

${ }^{23}$ J. A. Osborn, Phys. Rev. 67, 351 (1945).

${ }^{24}$ D. C. Worledge, Appl. Phys. Lett. 84, 4559 (2004).

${ }^{25}$ H. N. Pham, I. Dumitru, A. Stancu, and L. Spinu, J. Appl. Phys. 97, $10 \mathrm{P} 106$ (2005)

${ }^{26}$ B. N. Engel et al., IEEE Trans. Magn. 41, 132 (2005).

${ }^{27}$ W. J. Gallagher and S. S. P. Parkin, IBM J. Res. Dev. 50, 5 (2006).
${ }^{28}$ R. Urban, G. Woltersdorf, and B. Heinrich, Phys. Rev. Lett. 87, 217204 (2001).

${ }^{29}$ Y. Tserkovnyak, A. Brataas, and G. E. W. Bauer, Phys. Rev. Lett. 88, 117601 (2002).

${ }^{30}$ P. M. Braganca, I. N. Krivorotov, O. Ozatay, A. G. F. Garcia, N. C. Emley, J. C. Sankey, D. C. Ralph, and R. A. Buhrman, Appl. Phys. Lett. 87, 112507 (2005). 